\title{
Dye Sensitized Solar Cells Principles and New Design
}

\author{
Yang Jiao, Fan Zhang and Sheng Meng \\ Beijing National Laboratory for Condensed Matter Physics and \\ Institute of Physics, Chinese Academy of Sciences, Beijing
}

China

\section{Introduction}

It is generally believed that fossil fuels, the current primary but limited energy resources, will be replaced by cleaner and cheaper renewable energy sources for compelling environmental and economic challenges in the 21st century. Solar energy with its unlimited quantity is expected to be one of the most promising alternative energy sources in the future. Devices with low manufacturing cost and high efficiency are therefore a necessity for sunlight capture and light-to-energy conversion.

The dye-sensitized solar cell (DSSC), invented by Professor M. Grätzel in 1991 (O'Regan \& Grätzel, 1991), is a most promising inexpensive route toward sunlight harvesting. DSSC uses dye molecules adsorbed on the nanocrystalline oxide semiconductors such as $\mathrm{TiO}_{2}$ to collect sunlight. Therefore the light absorption (by dyes) and charge collection processes (by semiconductors) are separated, mimicking the natural light harvest in photosynthesis. It enables us to use very cheap, wide band-gap oxide semiconductors in solar cells, instead of expensive Si or III-V group semiconductors. As a result, much cheaper solar energy at $\$ 1$ or less per peak Watt $(\$ 1 / \mathrm{pW})$ can be achieved. For comparison, the dominant crystalline or thin-film Si solar cells have a price of $>\$ 4-5 / \mathrm{pW}$ presently and are suffering from the worldwide Si shortage. The fabrication energy for a DSSC is also significantly lower, $40 \%$ of that for a Si cell.

In this book chapter, we will present the principles of DSSC and detail the materials employed in a DSSC device in section 2. In section 3, the fabrication processes are shown. Then we discuss the energy conversion mechanism at the microscopic level in section 4 . After this we try to give new design of the dye molecule and adsorption anchoring configurations to give hints on improving the energy conversion efficiency and making more stable devices in section 5. At last we present our conclusion and perspectives.

\section{Principles of dye sensitized solar cells}

\subsection{Components}

The current DSSC design involves a set of different layers of components stacked in serial, including glass substrate, transparent conducting layer, $\mathrm{TiO}_{2}$ nanoparticles, dyes, electrolyte, and counter electrode covered with sealing gasket. The typical configuration is shown in Fig. 1. 

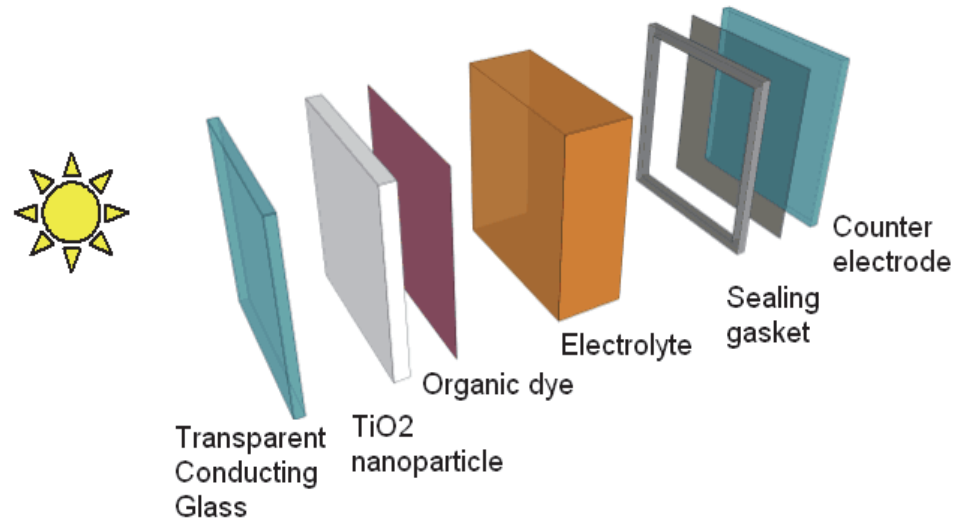

Fig. 1. Typical configuration of a DSSC.

\subsubsection{Transparent conducting glass}

In the front of the DSSC there is a layer of glass substrate, on top of which covers a thin layer of transparent conducting layer. This layer is crucial since it allows sunlight penetrating into the cell while conducting electron carriers to outer circuit. Transparent Conductive Oxide (TCO) substrates are adopted, including F-doped or In-doped tin oxide (FTO or ITO) and Aluminum-doped zinc oxide (AZO), which satisfy both requirements. ITO performs best among all TCO substrates. However, because ITO contains rare, toxic and expensive metal materials, some research groups replace ITO with FTO. AZO thin films are also widely studied because the materials are cheap, nontoxic and easy to obtain. The properties of typical types of ITO and FTO from some renowned manufacturers are shown in Table 1.

\begin{tabular}{|c|c|c|c|c|c|}
\hline $\begin{array}{c}\text { Conductive } \\
\text { glass }\end{array}$ & Company & $\begin{array}{c}\text { Light } \\
\text { transmittance }\end{array}$ & $\begin{array}{c}\text { Conductivity } \\
(\mathrm{Ohm} / \mathrm{sq})\end{array}$ & $\begin{array}{c}\text { Thickness } \\
(\mathrm{mm})\end{array}$ & $\begin{array}{c}\text { Size } \\
(\mathrm{cm} \times \mathrm{cm})\end{array}$ \\
\hline ITO & Nanocs & $>85 \%$ & 5 & 1.1 & $1 \times 3$ \\
\hline ITO & PG\&O & $85 \%$ & 4.5 & 1.1 & $2 \times 3$ \\
\hline FTO & NSG & $>84 \%$ & $<7$ & 3 & $100 \times 100$ \\
\hline
\end{tabular}

Table 1. Properties of a few types of commercial ITO and FTO materials.

\subsection{2 $\mathrm{TiO}_{2}$ nanoparticles}

DSSC has a low efficiency less than 1\% until Professor Grätzel employs porous $\mathrm{TiO}_{2}$ as the anode material. Usually a layer of negatively doped $\mathrm{TiO}_{2}\left(\mathrm{n}-\mathrm{TiO}_{2}\right)$ nanoparticles is used. The advantages of $\mathrm{TiO}_{2}$ include high photosensitivity, high structure stability under solar irradiation and in solutions, and low cost. The typical particle size is $8-30 \mathrm{~nm}$ in diameter, and the $\mathrm{TiO}_{2}$ films thickness is $2-20 \mu \mathrm{m}$, with the maximum efficiency located at a thickness of 12-14 $\mu \mathrm{m}$ depending on dyes and electrolyte chosen (Ito et al., 2008). However, as a wide bandgap semiconductor $(\sim 3.2 \mathrm{eV}), \mathrm{TiO}_{2}$ absorbs only UV light, which comprises only a small fraction $(\sim 5 \%)$ of solar spectrum. As a result, dye molecules are employed for visible light capture. Only nanocrystalline $\mathrm{TiO}_{2}$ provides high light capture efficiency, with external 
quantum efficiency (incident photon-to-charge efficiency) typically in the range of $60-90 \%$ using nanocrystal forms in comparison with $<0.13 \%$ using the monocrystal form (Grätzel, 2005). The reason lies in the high surface-to-volume ratios for porous nanocrystal materials.

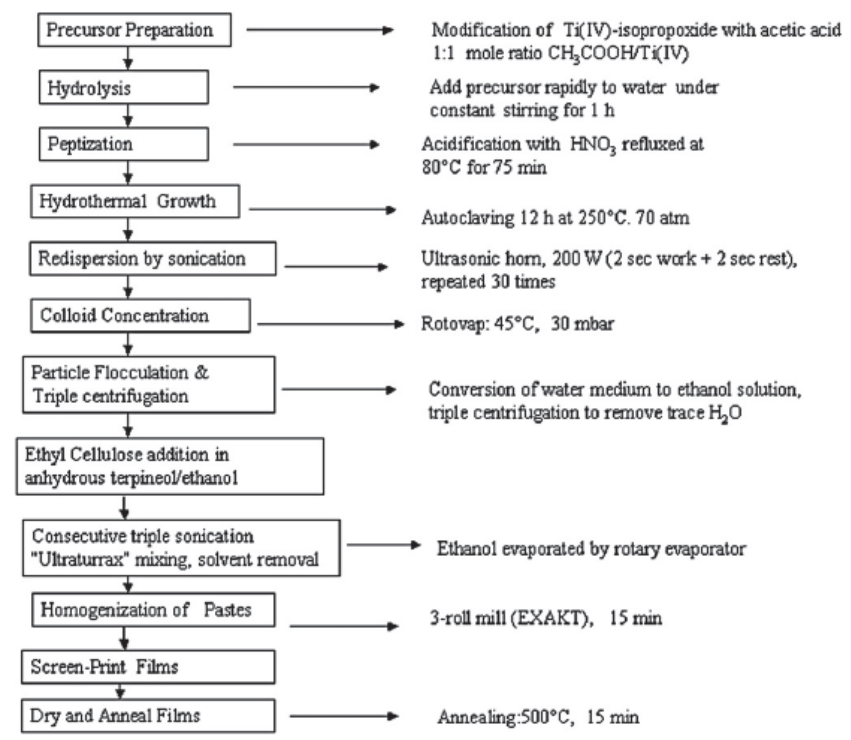

Scheme 1. Flow diagram depicting preparation of $\mathrm{TiO}_{2}$ colloid and paste used in screenprinting technique for DSSC production. Adopted from (Ito et al., 2008). Copyright: 2007 Elsevier B. V.

\subsubsection{Dyes}

Dye molecules are the key component of a DSSC to have an increased efficiency through their abilities to absorb visible light photons. Early DSSC designs involved transition metal coordinated compounds (e.g., ruthenium polypyridyl complexes) as sensitizers because of their strong visible absorption, long excitation lifetime and efficient metal-to-ligand charge transfer (O'Regan \& Grätzel, 1991; Grätzel, 2005; Ito et al., 2008). However, high cost of Ru dyes $(>\$ 1,000 / \mathrm{g})$ is one important factor hindering the large-scale implementation of DSSC. Although highly effective, with current maximum efficiency of 11\% (Grätzel, 2005), the costly synthesis and undesired environmental impact of those prototypes call for cheaper, simpler, and safer dyes as alternatives.

Organic dyes, including natural pigments and synthetic organic dyes, have a donor-acceptor structure called as push-pull architecture, thus improving short circuit current density by improving the absorption in red and infrared region. Natural pigments, like chlorophyll, carotene, and anthocyanin, are freely available in plant leaves, flowers, and fruits and fulfill these requirements. Experimentally, natural-dye sensitized $\mathrm{TiO}_{2}$ solar cells have reached an efficiency of $7.1 \%$ and high stability (Campbell et al., 2007).

Even more promising is the synthetic organic dyes. Various types have recently been developed, including indolic dyes (D102, D149) (Konno et al., 2007), and cyanoacrylic acids (JK, C209). The same as some natural dyes, they are not associated with any metal ions, 
being environmental benign and easily synthesized from abundant resources on a large scale. The efficiency has reached a high level of 10.0-10.3\% (Zeng et al., 2010). They are relatively cheap, at the cost of one-tenth of corresponding $\mathrm{Ru}$ dyes. Light soaking experiments have confirmed they possess long-time stability: $80 \%$ efficiency has been maintained after 1,200 hours of light-soaking at $60{ }^{\circ} \mathrm{C}(\sim 5$ million turnovers). The commercialized production of these synthetic dyes has been established in China this year. A single dye usually has a limited adsorption spectrum, so some research groups use several kinds of dyes to relay energy transfer and compensate each other and have achieved good results (Hardin et al., 2010).

\subsubsection{Electrolyte}

Currently three different kinds of electrolytes have been used in real DSSCs with pros and cons of each kind: (i) the most common electrolyte is $\mathrm{I}^{-} / \mathrm{I}_{3^{-}}$in organic solvents, such as acetonitrile. Sometimes lithium ion is added to facilitate electron transport. This kind of electrolyte is good for ion diffusion and infiltrate well with $\mathrm{TiO}_{2}$ film, keeping highest efficiency of all DSSCs. But limited long-term stability due to volatilization of liquid hinders its wide use. (ii) Inorganic ionic liquids made of salts or salt mixture. It looks like solid while it has properties of liquid and it performs well in conductivity. But after a long period of time, its efficiency declines. (iii) Solid electrolyte, such as spiro-MeOTAD or CuI (Konno et al., 2007). For CuI, its instability and crystallization makes it hard to fill in the porous $\mathrm{TiO}_{2}$ films. The problem can be solved by adding ionic liquid into the electrolyte. Spiro-MeOTAD is a typical kind of organic hole conductor, which has been developed for years and the DSSC based on this kind of electrolyte has reached the efficiency of 5\% (Yu et al., 2009).

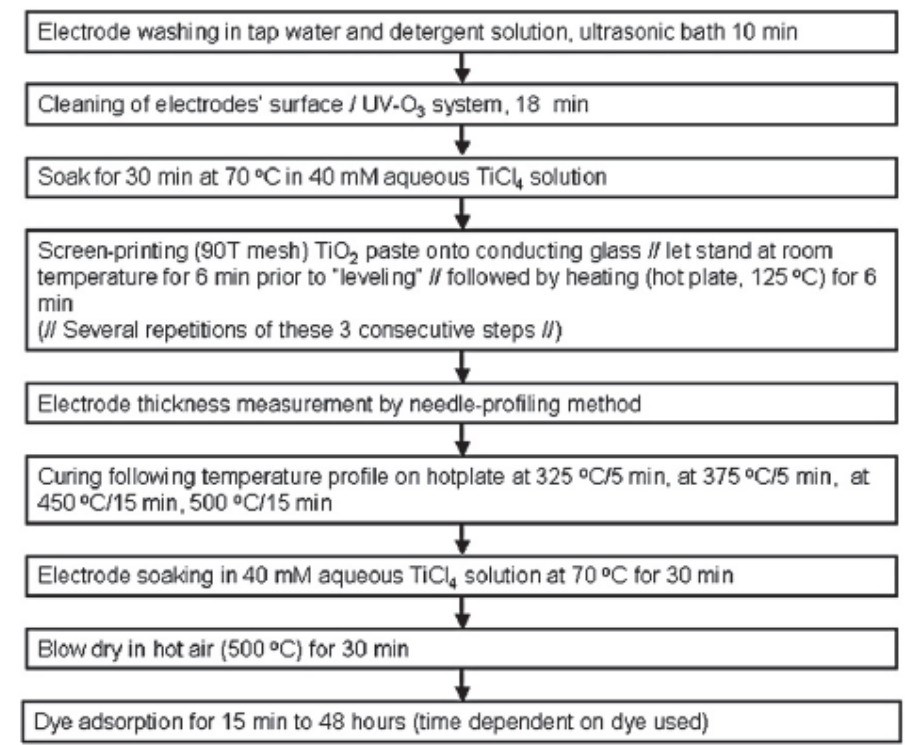

Scheme 2. Schematic representation for fabrication of dye-sensitized- $\mathrm{TiO}_{2}$ electrodes. Adopted from (Ito et al., 2008). Copyright: 2007 Elsevier B. V. 


\subsubsection{Counter electrode}

On the back of the DSSC there presents another glass substrate covered with a thin layer of Pt used as the catalyst to regenerate I- and as the cathode material. Pt is the best material to make efficient devices technically. But considering high expenses, carbon cathode has been an ideal substitute, such as carbon black, carbon nanotubes etc. In 2006, Grätzel's group employs carbon black as the material of counter electrode, and reaches an efficiency of $9.1 \%$, which is $83 \%$ of that using Pt (Yu et al., 2009).

Conducting polymers can also be used. Polyaniline film on stainless steel by electrochemical polymerization bas been reported as a counter electrode of DSSC (Qin et al., 2010). It is cheap and non-fragile.

\subsection{Fabrication}

In this section, we introduce Grätzel's new fabrication technologies for DSSCs having a conversion efficiency of solar light to electricity power over $10 \%$ (Ito et al., 2008). It consists of pre-treatment of the working photoelectrode by $\mathrm{TiCl}_{4}$, variations in layer thickness of the transparent nanocrystalline- $\mathrm{TiO}_{2}$ and applying a topcoat light-scattering layer as well as the adhesion of an anti-reflecting film to the electrode's surface.

First, one prepares glass substrate with a transparent conducting layer. Then, the DSSC working electrodes are prepared as shown in Scheme $1 \& 2$. Scheme 1 depicts procedures to produce paste (A) containing $20 \mathrm{~nm}$ nanocrystalline $\mathrm{TiO}_{2}$ particles. For the paste used in the light-scattering layers (paste $\mathrm{B}$ ), $10 \mathrm{~nm} \mathrm{TiO}_{2}$ particles which were obtained following the peptization step and in a procedure analogous to those of $20 \mathrm{~nm} \mathrm{TiO}$ outlined in Scheme 1, were mixed with $400 \mathrm{~nm} \mathrm{TiO}$ colloidal solution. Paste A and B are coated on FTO, forming the $\mathrm{TiO}_{2}$ "double layer" film. It is treated with $\mathrm{TiCl}_{4}$ before sintering. After cooling, the electrode is immersed in dye solutions.

Finishing fabrication of all parts, the dye-covered $\mathrm{TiO}_{2}$ electrode and Pt-counter electrode are assembled into a sandwich type cell and sealed with a hot-melt gasket. For the counter electrode, a hole (1-mm diameter) is drilled in the FTO glass. The hole is made to let the electrolyte in via vacuum backfilling. After the injection of electrolyte, the hole is sealed using a hot-melt ionomer film and a cover glass as shown in Fig. 2.

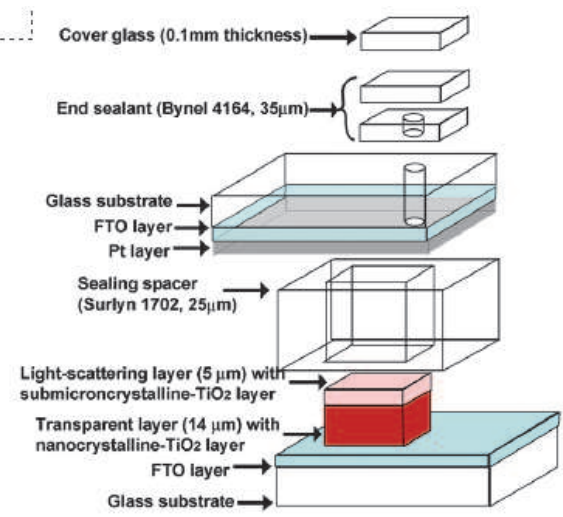

Fig. 2. Fabrication of the dye sensitized solar cells. Adopted from (Ito et al., 2008). Copyright: 2007 Elsevier B. V. 


\section{Processes}

The way that DSSC works is quite simple, shown by the scheme in Fig. 3 . The central idea is to separate the light absorption process from the charge collection process, mimicking natural light harvesting procedures in photosynthesis, by combining dye sensitizers with semiconductors. For most DSSC devices, the $\mathrm{I}^{-} / \mathrm{I}_{3^{-}}$couple is often used as a redox shuttle.

\subsection{Sunlight absorption and electronic excitation}

Photons of different energy in sunlight strike on the cell, penetrating into the dye layer since both the ITO layer with glass substrate and the $\mathrm{TiO}_{2}$ nanocrystals are transparent to visible light. If photon energy is close to the energy gap of the dye molecule, namely, the energy difference between the highest occupied molecular orbital (HOMO) and lowest unoccupied molecular orbital (LUMO), it will be absorbed by the dye, promoting one electron from HOMO to LUMO. To be effective, usually it requires HOMO of the dye to reside in the bandgap of the semiconductor, and the LUMO to lie within the conduction band of the semiconductor. But this is not always true. Meng and collaborators have shown that electrons can be injected from cyanin dyes to the $\mathrm{TiO}_{2}$ nanowire within $50 \mathrm{fs}$ after excitation, although the dye LUMO is originally lower than the $\mathrm{TiO}_{2}$ conduction band minimum (CBM) by $0.1-0.3 \mathrm{eV}$ (Meng et al., 2008). This extends the current understanding of the mechanisms of DSSC operation and thus can enhance the ability to optimize their design and efficiency.

\section{Dye Solar Cell Structure}

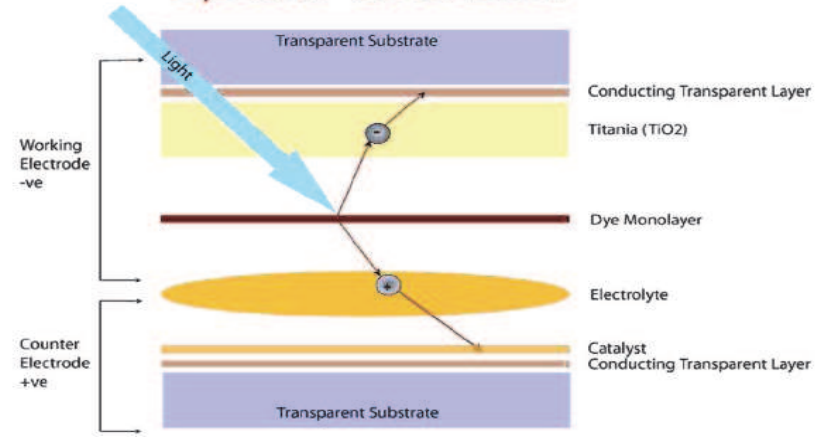

Fig. 3. Schematic illustration of the operation principle for molecular photovoltaic cell. Adopted from (Grätzel, 2009) Copyright: 1991 Nature Publishing Group.

\subsection{Electron-hole separation, electron injection and collection}

The excited electron will then inject into the conduction band of semiconductor through the interfacial bonds between the dye and the $\mathrm{TiO}_{2}$, and then be transported and finally collected by the ITO electrode (the anode). On the other hand, the hole generated by photon excitation remains on the molecule during this process, since the HOMO of dye is separated from all other energy levels of the device.

\subsection{Redox reaction at dye/electrolyte interface, and at counter electrode}

There is no energy channels for the hole to diffuse into $\mathrm{TiO}_{2}$ photoanode. As a result, the hole is eventually filled up by electrons from electrolyte ions, which conduct current 
between the cathode and the dye molecule. At the same time, reduction of oxidized dye by iodide produces triiodide. The triiodide diffuses to counter electrode and accepts electrons from external load, regenerating the iodides. The overall process will provide electron flow from the ITO side (anode) to the outer circuit, with the potential difference equals to the incident photon energy if no voltage loss occurs during electron injection, diffusion and the neutral dye regeneration. In reality, however, these processes are always present. So the anode-cathode potential difference, namely, the open-circuit voltage Voc, is mainly determined by the difference between conduction band bottom and electrolyte anion energy level, typically around 0.6-0.9 V.

\subsection{Recombination}

In all solar cells, the light generated carriers have a probability to meet their conjugate carriers and recombine. Recombination reduces the output of electrical power in DSSC. The photo injected electrons in the $\mathrm{TiO}_{2}$ have two possible recombination pathways: direct recombination with the oxidized dyes or with the $\mathrm{I}_{3}$ - in the electrolyte (Haque et al., 2005), which are shown in Fig. 4. The latter process is dominant and has been thoroughly studied. It can be reduced by adding a thin $\mathrm{TiO}_{2}$ under-layer between the FTO and the nanocrystalline porous $\mathrm{TiO}_{2}$ layer, because the connection between the FTO and the electrolyte is an important recombination route (Liu et al., 2006).

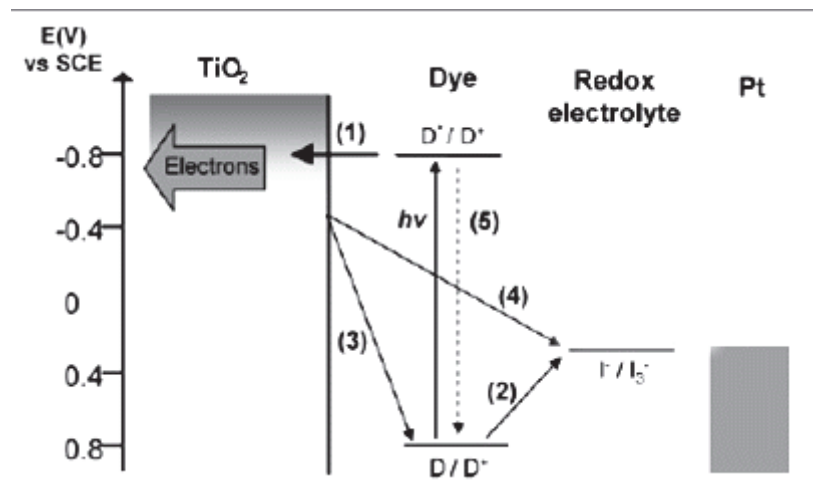

Fig. 4. Different electron transfer processes in the solar cell: (1) electron injection from dye excited state into the conduction band of $\mathrm{TiO}_{2}$ semiconductor; (2) regeneration of the dye cation by electron transfer from the redox couple; (3) charge recombination to the cation of the dye; (4) recombination to the redox couple; and (5) excited state decay to the ground state. Adopted from (Haque et al., 2005), Copyright: 2005 American Chemical Society.

\section{Electron dynamics in DSSC}

A DSSC benefits from its imitation of natural photosynthesis in that it separates sunlight absorption--which requires a large space--from electron collection processes which need highly pure materials and being most efficient on a small lengthscale. The biggest challenge to develop DSSCs is to realize both functions in the same system and to improve efficiency on both sides. By combining dye sensitizers with oxide semiconductor nanoparticles, DSSCs resolve this conflict. Visible light absorption efficiency is improved by $>1000$ times on 
nanoparticles compared to that of single-crystal surfaces, due to high surface/volume ratio of the former (Grätzel, 2005). Nevertheless, the mechanism and detailed dynamics of electron-hole separation at the dye/semiconductor interface, especially on a microscopic scale, remains elusive.

Experimentally it is observed that at various dye/ $\mathrm{TiO}_{2}$ interface, the timescale of excited electron injection ranges from the shortest $3 \mathrm{fs}$ for biisonicontinic acid in vacuum to $100 \mathrm{ps}$ for the triplet state injection of Ru-complex N719 in devices (see, for example, the summary in Meng \& Kaxiras, 2010). The huge time span suggests rich physical factors may play a role. Understanding this process will help us tune charge transfer timescale and improve sunlight-to-electricity conversion efficiency. For instance, electron injection will be 3.3 times slower with the addition of a $\mathrm{CH}_{2}$ group inserted between the dye molecule and the semiconductor, predicted from exponential decay of tunneling electron density when increasing separation distance in a non-adiabatic process. This is indeed observed in experiments using Re dyes (ReC1A-ReC3A) (Asbury et al., 2000). However, this pronounced time increase is not observed in experiments on Zn-porphyrins with one or four oligo(phenylethylny) bridges (Chang et al., 2009).

Meng et al. examined the influence of various factors on the electron injection efficiency using state-of-the-art first-principles calculations within the framework of time-dependent density functional theory (TDDFT). From TDDFT simulations, they found that among the three organic dyes they investigated longer molecules do involve a longer injection time, which is consistent with intuition (Meng \& Kaxiras, 2010). Furthermore, the time elongation is only 1.2 times (by inserting a $(\mathrm{CH})_{2}$ group) or 1.3 times (inserting a thiophene group). This indicates that adiabatic processes play a major role in these cases, which also explains the Zn-porphyrin experiment. Figure 5 shows the structure of the three organic dyes and their absorption intensity in neutral and deprotonated states.

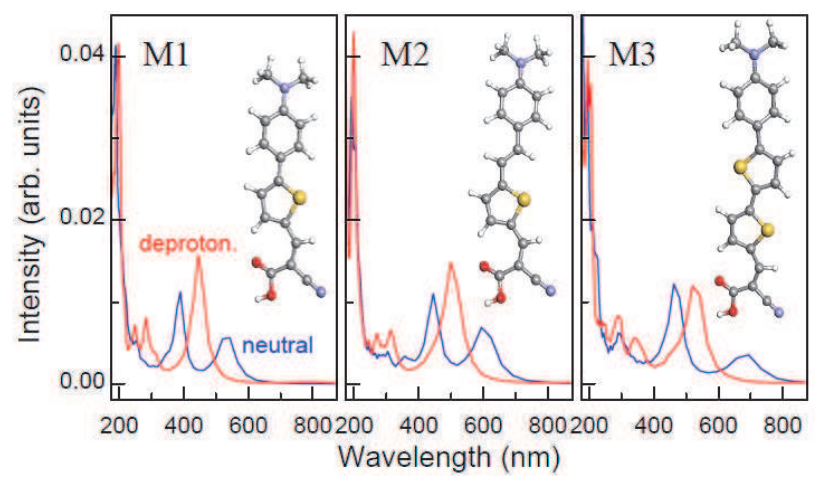

Fig. 5. Structure of the three organic dyes investigated and their absorption spectra. Blue lines: neutral dye; Red: deprotonated dyes. Adopted from (Meng \& Kaxiras, 2010). Copyright: 2010 American Chemical Society.

On the basis of a previous work which illustrates an ultrafast electron-hole separation process at the anthocyanin $/ \mathrm{TiO}_{2}$ interface (Meng et al., 2008), they extended to demonstrate further that various factors including dye molecular size, binding geometry, and point defects on the $\mathrm{TiO}_{2}$ surface will greatly influence electron collection efficiency. To analyze 
quantitatively the charge transfer and electron-hole separation process, they use the integral of excited electron (hole) density projected onto the $\mathrm{TiO}_{2}$ orbitals, $\chi$, as a function of time after photon absorption, with $\chi$ defined as

$$
\chi=\int d r|\psi(r)|^{2} \quad \psi(r)=\sum_{j \in T i O_{2}} c_{j} \varphi_{j}(r)
$$

where $c_{j}$ are the coefficients of atomic basis states $\varphi_{j}$ in the Kohn-Sham orbital $\psi_{\kappa s}(r)$ of interest ( either the excited electron or the hole)

$$
\psi_{K S}(r)=\sum_{j} c_{j} \varphi_{j}(r)
$$

The curves in Fig. 6 show the injection probability, $\chi$, of the excited electron and hole as a function of simulation time.
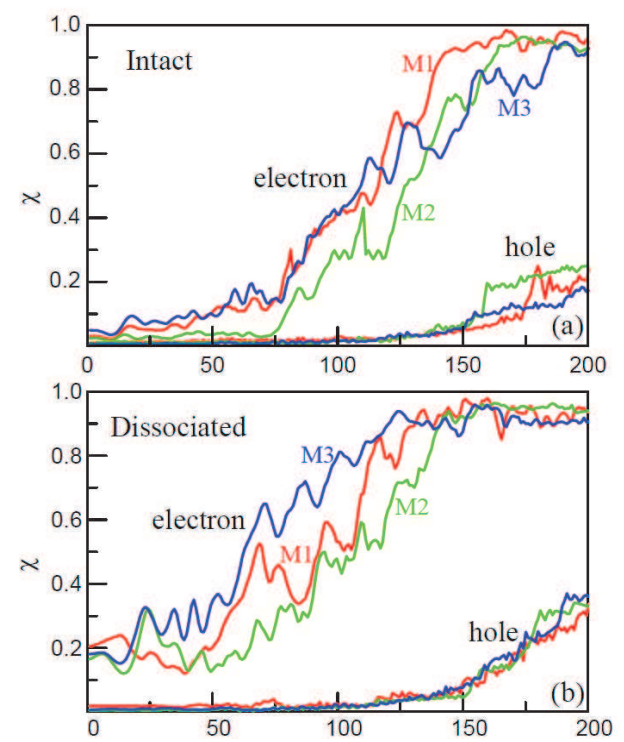

Fig. 6. Electron-hole dynamics from TDDFT simulations. Vertical axis demonstrates electrons injected into $\mathrm{TiO}_{2}$, horizontal axis denotes time simulated. Adopted from ( Meng \& Kaxiras, 2010 ). Copyright: 2010 American Chemical Society

They also found that dye adsorption configurations significantly affect electron injection. By comparing to measured spectroscopy, intact and dissociative dye adsorptions are identified. The former is $30-50 \mathrm{fs}$ slower than the latter. Different adsorption configurations of intact dyes result in injection time varies by three folds. The difference is mainly caused by the interface dipole moments. A positive dipole at the interface introduces an upshift of CBM, which will suppress excited electron transfer from the dye molecule to semiconductor conduction bands. 
The semiconductor surface also imposes a fundamental influence on electron-hole dynamics. Dye adsorption on surface oxygen vacancies is very stable; it leads to a strong electronic coupling between the dye and the surface resulting in an electron injection time of $\sim 50 \mathrm{fs}, 2-3$ times faster than that on defect-free surfaces. But this improvement is at the cost of fast electron-hole recombination, which will reduce device efficiency. These simulations could explain well the two injection times at $40 \mathrm{fs}$ and $200 \mathrm{fs}$ observed in experiments, which would correspond to adsorption on defects and on clean surfaces, respectively.

They also studied electron back transfer after the excited electron has been completely injected into the $\mathrm{TiO}_{2}$. All the research results indicate that electrons and holes are separated in space at a time $\sim 200 \mathrm{fs}$, to assure DSSCs work well.

\section{New design}

Recent developments of effective all-organic dyes have introduced a novel Donor-חAcceptor (D-ח-A) structure, with the donor and acceptor connected via a molecular bridge conductor (n-group) and the acceptor directly bound to semiconductor surface. Upon excitation, electrons in the HOMOs, originally distributed around the donor group, are promoted to the LUMOs, which are centred around the acceptor. This excitation process effectively pushes extra electrons to the acceptor part of the dyes and leaves holes on the donor part. Importantly, the proximity between the acceptor and the semiconductor surface, mutually bound via covalent and/or hydrogen bonds, allows ultrafast transfer of excited electrons to the conduction bands of the semiconductor (Meng et al., 2008; Duncan \& Prezhdo, 2007). Consequently, this leads to efficient electron-hole separation and ultimately electricity generation.

Laboratory development of D-ח-A dyes often invokes a trial-and-error approach, which requires extensive chemical synthesis and expensive materials processing with a slow progress. In this regard, theoretical screening of potential organic dyes using state-of-the-art first principles computation shows a great promise, significantly reducing the cost to develop efficient dyes and expediting discovery of new ones.

\subsection{Enhanced light absorption}

Based on first principles calculations on the electronic structure and optical absorption of organic molecules, we design a set of Donor-ח-Acceptor (D-ח-A) type of dyes with inserting and changing intermediate spacer groups or electron acceptor groups, to be used in DSSCs for sunlight harvesting and energy conversion. We found that with these modifications, the electronic levels and corresponding optical absorption properties of organic dyes can be gradually tuned, with dyes having novel 1,4-cyclohexadiene groups as promising candidates for red light absorption and achieving high extinction coefficients. This study opens a way for material design of new dyes with target properties for further optimizing the performance of dye solar cells.

Our calculations are performed within the framework of DFT (Kohn \& Sham, 1965) (for structure optimization) and TDDFT (Runge \& Gross, 1984) (for excited states and optical absorption), using SIESTA (Soler et al., 2002) and Gaussian (Frisch et al., 2009) packages. It is well known that popular functionals (such as PBE and B3LYP) systematically underestimate charge-transfer excitation energies and result optical band splitting, leading to problematic predictions on optical excitations. Most recently, significant progresses have been made by including self-interaction correlations at the long range in hybrid fuctionals, to properly 
account for the long-range charge transfer excitation while retain the reliable description of short-range correlation interactions. Our previous work shows that the long-range corrected (LC) functionals $\omega$ B97X (Chai \& Head-Gordon, 2008) and CAM-B3LYP (Yanai et al., 2004) ) yield better comparison with experiment. Since $\omega$ B97X has less parameters and shows better agreement with experimental results (after solvent effect correction of 0.1-0.3 eV (Pastore et al., 2010)), we use this functional in the following for predicting optical properties of new dyes.

For structure optimization, we use pseudopotentials of the Troullier-Martins type to model the atomic cores, the PBE form of the exchange-correlation functional, and a local basis set of double- $\zeta$ polarized orbitals. An auxiliary real space grid equivalent to a planewave cutoff of $680 \mathrm{eV}$ is used for the calculation of the electrostatic (Hartree) term. A molecular structure is considered fully relaxed when the magnitude of forces on the atoms is smaller than $0.03 \mathrm{eV} / \AA$. Optical absorption are extracted from TDDFT simulations within the linear response regime at fixed dye geometry in gas phase. We use the 6-31G(d) basis set throughout this book chapter, which has been shown to yield negligible difference in electron density and energy accuracy compared with those including additional diffuse functions (Pastore et al., 2010). Within TDDFT, different functionals based on adiabatic approximation are used, including B3LYP and $\omega$ B97X. The latter one, belonging to LC functionals, are employed to correct excitation energies for charge-transfer excitations, which prevail in these dyes. The spectrum is obtained using the following formula based on calculated excitation energies and oscillator strengths,

$$
\varepsilon(\omega)=2.174 \times 10^{8} \sum_{I} \frac{f_{I}}{\Delta} \exp \left[-2.773 \frac{\left(\omega-\omega_{I}\right)^{2}}{\Delta^{2}}\right],
$$

where $\varepsilon$ is the molar extinction coefficient given in unit of $\mathrm{M}^{-1} \mathrm{~cm}^{-1}$, energies and $\omega_{I}$ are in unit of $\mathrm{cm}^{-1}$ and $f_{I}$ is the corresponding oscillator strength. Such a formula will satisfy the well-known relationship

$$
4.32 \times 10^{-9} \int \varepsilon(\omega) d \omega=\sum_{\mathrm{I}} f_{I} .
$$

We use a gaussian full-bandwidth of $\Delta=3000 \mathrm{~cm}^{-1}$ at half-height for optical band broadening to mimic thermodynamic oscillations in dye structures and excitations at room temperature in vacuum.

Organic dyes with the cyanoacrylic acid anchoring and electron with-drawing group have been very successful in real devices. We choose Y-1, Y-2 and Y-3 molecules (left column, Fig. 7) as the model dyes because they have a simple structure and have been successfully synthesized for DSSC applications (for example, as a1, b1, b2 dyes in reference Kitamura et al., 2004). Then the model dyes are modified by inserting a cyclohexadene in the $C=C$ double bond on the backbone. The resulting dyes are named as dyes Y-1ben, Y-2ben, Y-3ben hereafter for convenience. Another model dye Y-1ben2 is constructed by inserting an additional cyclohexadene ring in the Y-1ben (right column, Fig. 7). All these dyes have a strong structural similarity with each other; we hope upon these variations in the dye structures, various aspects of the atomic structure, electronic structure, absorption character and therefore the structure-property relationship of organic dyes can be investigated. 
The calculated optical absorption spectra are shown in Fig. 8. We found that the absorption spectra will be red shifted by prolonging the oligoene backbone (compare the green and red lines) and inserting cyclohexadiene moiety (compare the lines in the same colour). Attaching benzene rings to the amide nitrogen could enhance the absorption intensity a little but barely changes the position of maximum absorption (compare blue and green lines). On the other hand, inserting cyclohexadiene group could modify the spectra significantly, both in peak positions and intensity (compare the lines in Fig. 8 in the same colour). This would make dyes with cyclohexadiene group attractive candidates for future development of DSSC devices, especially for high extinction, long wavelength light absorption.

We analyse further the electronic energy level calculated using B3LYP/6-31G(d) and the first excitation energy from $\omega \mathrm{B} 97 \mathrm{X} / 6-31 \mathrm{G}(\mathrm{d})$ (Table 1). It is known that the solvent effects will lower dye absorption energy by $0.1-0.3 \mathrm{eV}$ (Pastore et al., 2010); therefore experimental values quoted in Table 2 which are measured in solution would have been corrected by 0.1 -

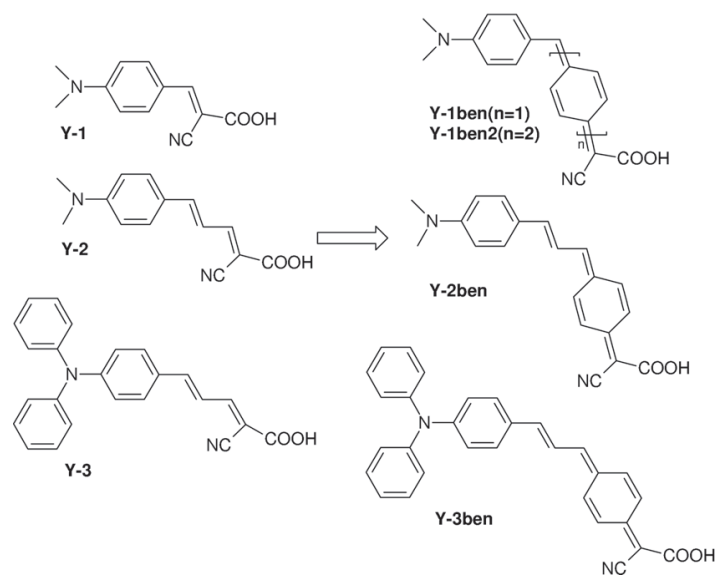

Fig. 7. Chemical structure of model dyes. The left column from up to bottom depicts $Y-1, Y-$ 2, Y-3, and the right column shows Y-1ben (Y-1ben2), Y-2ben, Y-3ben.

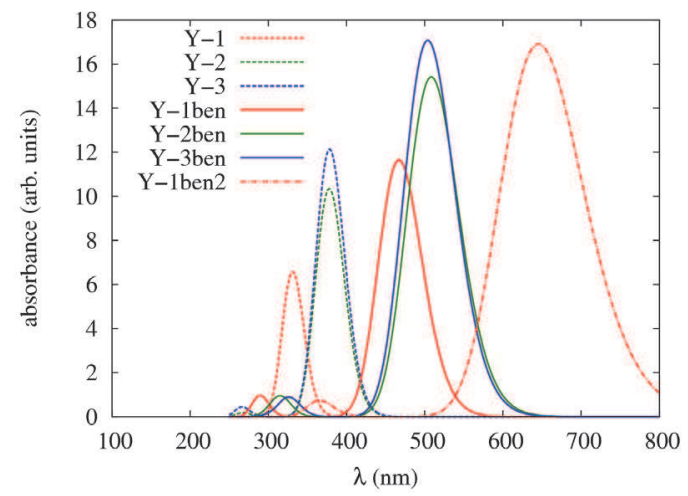

Fig. 8. Calculated optical absorption spectra of the model dyes. 
$0.3 \mathrm{eV}$, this leads to a better agreement with the results from LC-TDDFT for dyes in vacuum. For Y-1 dye the LC seems not necessary, maybe due to its short length.

Intuitively, the energy levels of the whole dye molecule are modified by the electric field introduced by the presence of chemical groups at the acceptor end. They would change according to the electronegativity of these chemical groups. The higher negativity, the lower the LUMO level, the smaller the energy gap would be. Our results proved this simple rule by showing that inserting electron withdrawing groups $\mathrm{C}=\mathrm{C}$ or cyclohexadiene will down shift the LUMO level and producing a smaller energy gap (Table 1).

Following this way we obtain a small energy gap of $1.41 \mathrm{eV}$ for Y-1ben2. In particular, we emphasize that the LUMO level is still higher by at least $0.7 \mathrm{eV}$ than the conduction band edge (CBE) of anatase $\mathrm{TiO}_{2}$, located at $-4.0 \mathrm{eV}$ (Kavan et al., 1996). This would provide enough driving force for ultrafast excited state electron injection. Another requisite in interface energy level alignment for DSSCs to work properly is that the dye HOMO has to be lower than the $\mathrm{I}-/ \mathrm{I}_{3}^{-}$redox potential at about $-4.8 \mathrm{eV}$. The potential level is comparable to cyclohexadiene dye HOMOs in Table 2. However, we note here that by comparing calculated energy levels with experimental measured potentials, the HOMO and LUMO positions are generally overestimated by $\sim 0.6 \mathrm{eV}$ and $\sim 0.3 \mathrm{eV}$, respectively. We expect the same trend would occur for dyes discussed in the present work; taking this correction into account, the designed new dyes would have a perfect energy alignment with respect to the CBE of TiO2 (more than $0.4 \mathrm{eV}$ lower than LUMOs) and the $\mathrm{I}-/ \mathrm{I}_{3}^{-}$redox potential $(\sim 0.5-1 \mathrm{eV}$ higher than HOMOs) favouring DSSC applications.

\begin{tabular}{|l|l|l|l|l|l|l|l|}
\hline Dyes & Y-1 & Y-2 & Y-3 & Y-1ben & Y-2ben & Y-3ben & Y-1ben2 \\
\hline LUMO & -2.22 & -2.49 & -2.63 & -2.92 & -3.05 & -3.09 & -3.30 \\
\hline HOMO & -5.64 & -5.36 & -5.38 & -5.15 & -5.02 & -5.06 & -4.71 \\
\hline gap & 3.42 & 2.87 & 2.75 & 2.23 & 1.97 & 1.97 & 1.41 \\
\hline فB97X & 3.74 & 3.28 & 3.28 & 2.65 & 2.44 & 2.46 & 1.92 \\
\hline Exp. $^{\text {a }}$ & 3.28 & 3.01 & 2.98 & & & & \\
\hline
\end{tabular}

Table 2. Electronic and optical properties of dyes predicted from first-principles calculations. The LOMO, HOMO and gap are results using B3LYP exchange-correlation potential. $\omega$ B97X indicates the first excitation energy using the $\omega \mathrm{B} 97 \mathrm{X}$ long correction.

a The data are from reference (Kitamura et al., 2004). The first absorption peak observed at $1.6 \times 10^{-5} \mathrm{~mol} \bullet \mathrm{dm}^{-3}$ in ethanol. Energy levels are in unit of eV.

To further demonstrate the electronic properties for these promising dyes, we show the wavefunction plots for the molecular orbitals HOMO and LUMO of dye Y-1ben. The contour reveals that the HOMO and LUMO extend over the entire molecule. Other modified dyes have a similar characteristic.

We conclude that with the insertion of electron with-drawing groups $\mathrm{C}=\mathrm{C}$ and cyclohexadiene in the backbone of oligoene dyes, we can tune the dye electronic levels relative to $\mathrm{TiO}_{2}$ conduction bands and the corresponding optical absorption properties as shown in Fig. 8 and Table 2, for optical performance when used in real DSSC devices. In particular, we propose that the model dyes with cyclohexadiene group might be promising candidates for red to infrared light absorption, which may offer improved sunlight-toelectricity conversion efficiency when used alone or in combination with other dyes. The anchoring geometry will also influence the energy level alignment and energy conversion efficiency, which will be discussed in the next subsection. 

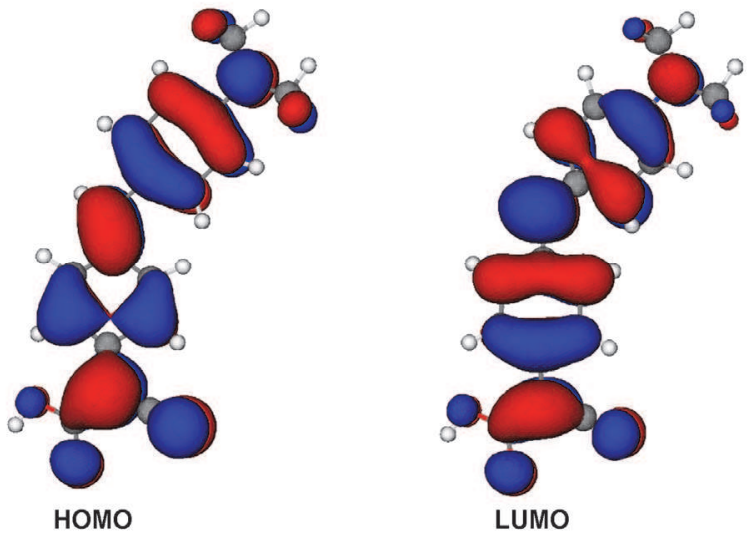

Fig. 9. Wavefunction plots for the representative dye Y-1ben. HOMO and LUMO orbitals are shown.

\subsection{Stable anchoring}

Following the similar principles, we have extended our study to the design of purely organic dyes with a novel acceptor, since the acceptor group connects the dye to the semiconductor and plays a critical role for dye anchoring and electron transfer processes. Our strategy is to test systematically the influence of chemical group substitution on the electronic and optical properties of the dyes.

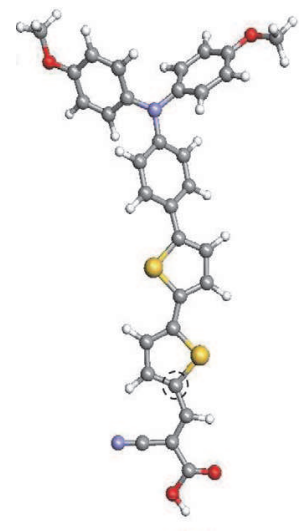

da1

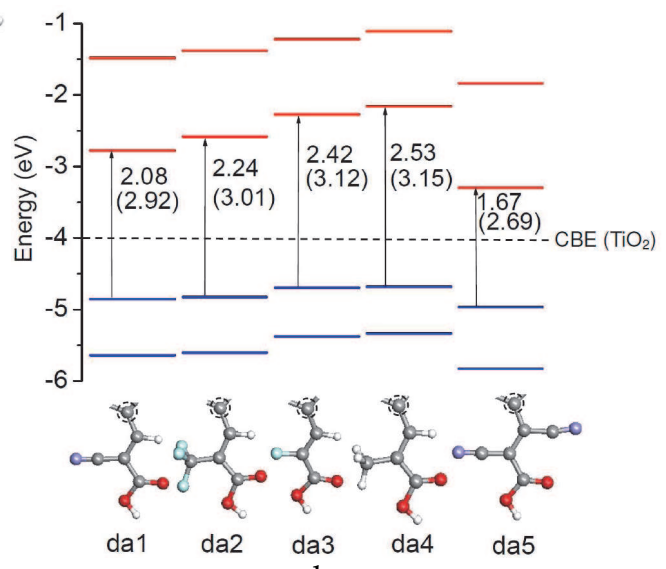

b

Fig. 10. (a) Optimized structure for the model dye da1. (b) The structure of acceptor groups by design and corresponding electronic level. The dashed circle marks the carbon atom connecting the acceptor part and the $\Pi$ bridge. Adapted from (Meng et al., 2011). Copyright: 2011 American Chemical Society.

Chemical groups of different electronegativity, size and shape and at different sites have been investigated. As an example, we consider here a specific modification on existing dye 
structures: replacement of the -CN group of cyanoacrylic side with other elements or groups. This gives a group of new dyes which we label da- $n$ (with $n=1-5$, an index). Organic dyes with the carboxylate-cyanoacrylic anchoring group have been very successful in real devices. From the point of view of electronic structure and optical absorption, it is possible that the side cyano group $(-\mathrm{CN})$ has a positive influence on light absorption and anchoring to the $\mathrm{TiO}_{2}$ surface (Meng et al., 2010). Accordingly, we consider the possibility of replacing $-\mathrm{CN}$ by other chemical groups and examine the dependence of dye performance on these groups. In Fig. 10 we show the set of dye acceptor structures we have investigated. We have replaced the cyano $-\mathrm{CN}$ group in model dye da1 by $-\mathrm{CF}_{3},-\mathrm{F}$, and $-\mathrm{CH}_{3}$ groups, which are labelled da2, da3, and da4, respectively. Model dye da1, shown in Fig. 10, has a very similar structure to that of D21L6 dye synthesized experimentally (Yum et al., 2009), except that the hexyl tails at the donor end are replaced by methyl groups. The electronic energy levels of these modified dyes in the ground-state are also shown in Fig. 10, as calculated using B3LYP/6-31G(d). Compared to the relatively small gap of $2.08 \mathrm{eV}$ for da1, the energy gap is increased by all these modifications. We have also tried many other groups, such as $-\mathrm{BH}_{2},-\mathrm{SiH}_{3}$, etc., for substituting the $-\mathrm{CN}$ group. All these changes give a larger energy gap. This may explain the optimal performance in experiment of the cyano $\mathrm{CN}$ group as a part of the molecular anchor, which yields the lowest excitation energy favouring enhanced visible light absorption. The changes in electronic structure introduced by substitution of the - $\mathrm{CN}$ group by other groups are a result of cooperative effects of both electronegativity and the size and shape of the substituted chemical groups.

We did not find any single-group substitution for the - $\mathrm{CN}$ that produces a lower energy gap. Therefore we extended our investigation to consider other possibilities. We found that with the substitution of the $-\mathrm{H}$ on the next site of the backbone by another $-\mathrm{CN}$ group, the ground-state energy gap is reduced to $1.67 \mathrm{eV}$ (see Fig. 10, dye da5). The LUMO level is higher than the conduction band edge of anatase $\mathrm{TiO}_{2}$ (dashed line, Fig. 10) and HOMO level is lower than the redox potential of tri-iodide, providing enough driving force for fast and efficient electron-hole separation at the dye $/ \mathrm{TiO}_{2}$ interface. With the above systematic modifications of the dye acceptor group, the electronic levels can be gradually tuned and as a consequence dyes with desirable electronic and optical properties can be identified. Influence of these acceptor groups on excited state electron injection can be studied in the same way as that in Section 4.

We also strive to design dye acceptors that render a higher stability of the dye $/ \mathrm{TiO}_{2}$ interface when used in outdoors applications. It was previously found that some organic dyes do not bind to the $\mathrm{TiO}_{2}$ photoanode strongly enough, and will come off during intensive light-soaking experiments, while other dyes show higher stability in such tests $(\mathrm{Xu}$ et al., 2009). Dye anchors with desirable binding abilities will contribute greatly to the stability of interface. A type of dye anchor under design is a cyano-benzoic acid group, whose binding configuration to the anatase $\mathrm{TiO}_{2}$ (101) surface is shown in Fig. 11. A particular advantage of cyano-benzoic acid as a dye acceptor is that, it strongly enhances dye binding onto $\mathrm{TiO}_{2}$ surfaces. We investigate the binding geometries of this organic dye on $\mathrm{TiO}_{2}$ (101) using DFT. Among several stable binding configurations, the one with a bidentate bond and a hydrogen bond between $-\mathrm{CN}$ and surface hydroxyl (originating from dissociated carboxyl acid upon adsorption), is the most stable with a binding energy of 1.52 $\mathrm{eV}$. The bond lengths are $\mathrm{d}_{\mathrm{Ti1}-\mathrm{O} 1}=2.146 \AA \mathrm{d}_{\mathrm{Ti2}-\mathrm{O} 2}=2.162 \AA$, and $\mathrm{d}_{\mathrm{CN} . . . \mathrm{HO}}=1.80 \AA$. Since there are three bonds formed, the dye is strongly stabilized on $\mathrm{TiO}_{2}$. Experimentally dyes with cyano-benzoic acid anchors have been successfully synthesized and the corresponding stability is under test (Katono et al., submitted). 


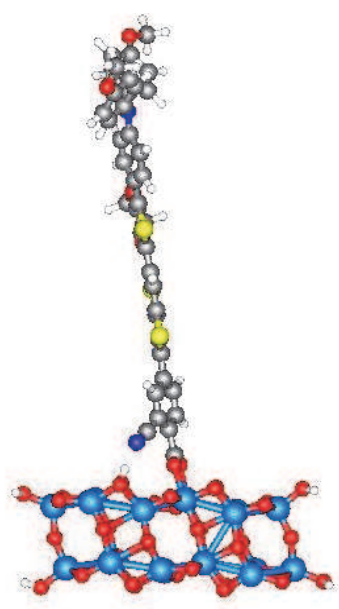

a

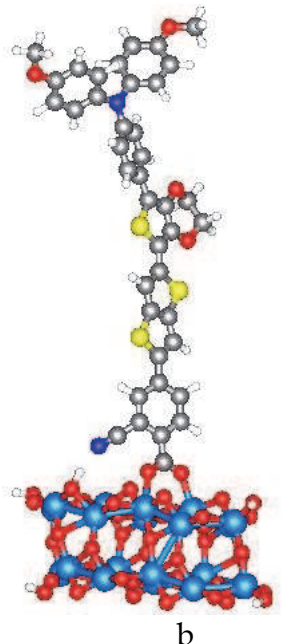

$\mathrm{b}$

Fig. 11. (a) Side and (b) front views of adsorption of the dye with a cyano-benzoic acid anchor on $\mathrm{TiO}_{2}$ nanoparticle.

\section{Conclusion}

In summary, after a brief introduction of the principles of DSSCs, including their major components, the fabrication procedures and recent developments, we try to focus on the atomic mechanisms of dye adsorption and electron transport in DSSCs as obtained from accurate quantum mechanical simulations. We discovered that electron injection dynamics is strongly influenced by various factors, such as dye species, molecular size, binding group, and surface defects; more importantly, the time scale for injection can be tuned by changing these parameters. Based on the knowledge about the interface electronic structure and dynamics at the molecular level, we strive to design new dye molecules and anchoring configurations employing state-of-the-art first principles calculations. Our results show that upon systematic modifications on the existing dye structures, the optical absorption and energy levels could be gradually tuned. In particular, we propose that by inserting cyclohexadiene groups into spacing $\mathrm{C}=\mathrm{C}$ double bonds, simple organic dyes could be promising candidates for enhanced red to infrared light absorption. This study opens a way for material design of new dyes with target properties to advance the performance of organic dye solar cells.

\section{Acknowledgment}

We thank our collaborators Professor Efthimios Kaxiras and Professor Michael Grätzel. This work is financially supported by NSFC (No. 11074287), and the hundred-talent program and knowledge innovation project of CAS.

\section{References}

Asbury, J. B.; Hao, E.; Wang, Y. \& Lian, T. (2000). Bridge Length-Dependent Ultrafast Electron Transfer from Re Polypyridyl Complexes to Nanocrystalline TiO2 Thin 
Films Studied by Femtosecond Infrared Spectroscopy. Journal of Physical Chemistry $B$ Vol 104, pp. 11957-11964, ISSN 1520-6106

Campbell, W. M.; Jolley, K. W.; Wagner, P.; Wagner, K.; Walsh, P. J.; Gordon, K. C.; SchmidtMende, L.; Nazeeruddin, M. K.; Wang, Q.; Grätzel, M. \& Officer, D. L. (2007). Highly Efficient Porphyrin Sensitizers for Dye-Sensitized Solar Cells, Journal of Physical Chemistry C, Vol. 111, No.32, (August 2007), pp. 11760-11762, ISSN 19327447

Chai, J.-D.; Head-Gordon, M. (2008). Systematic Optimization of Long-Range Corrected Hybrid Density Functionals, Journal of Chemical Physics, Vol. 128, No.8, (February 2008) pp.084106, ISSN 0021-9606

Chang, C. W.; Luo, L.; Chou, C. K.; Lo, C. F.; Lin, C. Y.; Hung, C. S.; Lee, Y. P. \& Diau, E. W. (2009). Femtosecond Transient Absorption of Zinc Porphyrins with Oligo(phenylethylnyl) Linkers in Solution and on TiO2 Films. Journal of Physical Chemistry C Vol 113, pp. 11524-11531, ISSN 1932-7447

Duncan, W. R.; Colleen, F. C.; Oleg, V. P. (2007). Time-Domain Ab Initio Study of Charge Relaxation and Recombination in Dye-Sensitized $\mathrm{TiO}_{2}$, Journal of the American Chemical Society, Vol.129, No.27, (June 2007) pp.8528-8543, ISSN 0002-7863

Frisch, M. J. et al. (2009). Gaussian 09, Revision A.1, Gaussian Inc.: Wallingford, CT, 2009

Grätzel, M. (2005). Mesoscopic Solar Cells for Electricity and Hydrogen Production from Sunlight, Chemistry Letters, Vol.34, No.1, (January 2005), pp. 8-13, ISSN 0366-7022

Grätzel, M. (2009). Recent Advances in Sensitized Mesoscopic Solar Cells, Accounts of Chemical Research, Vol.42, No.11, (November 2009), pp. 1788-1798, ISSN 0001-4842

Haque, S. A.; Palomares, E.; Cho, B. M.; Green, A. N. M.; Hirata, N.; Klug, D. R. \& Durrant, J. R. (2005). Charge Separation Versus Recombination in Dye-Sensitized Nanocrystalline Solar Cells: the Minimization of Kinetic Redundancy, Journal of the American Chemical Society, Vol.127, No.10, (March 2005), pp.3456-3462, ISSN 00027863

Hardin, B.E.; Yum, J.-H.; Hoke, E.T.; Jun, Y.C.; Pechy, P.; Torres, T.; Brongersma, M.L.; Nazeeruddin, M.K.; Graetzel, M.; \& McGehee, M.D. (2010). High Excitation Transfer Efficiency from Energy Relay Dyes in Dye-Sensitized Solar Cells, Nano Letters, Vol. 10, pp. 3077-3083, ISSN 1530-6984

Ito, S.; Murakami, T. N.; Comte, P.; Liska, P.; Grätzel, C.; Nazeeruddin, M. K. \& Grätzel, M. (2008). Fabrication of Thin Film Dye Sensitized Solar Cells With Solar to Electric Power Conversion Efficiency over 10\%, Thin Solid Films, Vol.516, No.14, (May 2008), pp.4613-4619, ISSN 0040-6090

Katono, M.; Bessho, T.; Meng, S.; Zakeeruddin, S. M.; Kaxiras, E.; Grätzel, M. (2011). Submitted.

Kavan, L.; Grätzel, M.; Gilbert, S. E.; Klemenz, C.; Scheel, H. J. (1996). Electrochemical and Photoelectrochemical Investigation of Single-Crystal Anatase, Journal of American Chemical Society. Vol. 118, No.28 (July 1996) pp. 6716-6723, ISSN 0002-7863

Kitamura, T.; Ikeda, M.; Shigaki, K.; Inoue, T.; Anderson, N. A.; Ai, X.; Lian, T. \& Yanagida, S. (2004). Phenyl-Conjugated Oligoene Sensitizers for TiO2 Solar Cells, Chemcal Materials, Vol.16, No.9, (February 2004) pp. 1806-1812, ISSN 0897-4756

Kohn, W.; Sham, L. J. (1965). Self-Consistent Equations Including Exchange and Correlation Effects. Physical Review, Vol.140, No.4A, (1965) pp. 1133-1138, ISSN 0031-899X

Konno, A.; Kumara, G. R. A.; Kaneko, S. (2007). Solid-state solar cells sensitized with indoline dye, Chemistry Letters, Vol.36, No.6, (June 2007), pp.716-717, ISSN 03667022 
Liu, X.; Huang, Z.;Meng, Q. et al. (2006). Recombination Reduction in Dye-Sensitized Solar Cells by Screen-Printed $\mathrm{TiO}_{2}$ Underlayers, Chinese Physics letters, Vol.23, No.9, (June 2006), pp.2606-2608, ISSN 0256-307X

Meng, S.; Ren, J. \& Kaxiras, E. (2008). Natural Dyes Adsorbed on TiO2 Nanowire for Photovoltaic Applicaitons: Enhanced Light Absorption and Ultrafast Electron Injection, Nano Letters, Vol.8, No.10, (September 2008), pp.3266-3272, ISSN 15306984

Meng, S. \& Kaxiras, E. (2010). Electron and Hole Dynamics in Dye-Sensitized Solar Cells : Influencing Factors and Systematic Trends, Nano Letters, Vol. 10, No.4 (April 2010), pp 1238-1247, ISSN 1530-6984

Meng, S.; Kaxiras, E; Nazeeruddin, Md. K. \& Grätzel, M. (2011). Design of Dye Acceptors for Photovoltaics from First-principles Calculations, Journal of Physical Chemistry C, in press, ISSN 1932-7447

O’Regan, B. \& Grätzel, M. (1991). A Low-Cost, High-Efficientcy Solar-Cell Based on DyeSensitized Colloidal $\mathrm{TiO}_{2}$ Films, Nature, Vol.353, No.6346, (October 1991), pp. 737740, ISSN 0028-0836

Pastore, M.; Mosconi, E,; De Angelis, F. \& Grätzel, M. (2010). A Computational Investigation of Organic Dyes for Dye-Sensitized Solar Cells: Benchmark, Strategies, and Open Issues, Journal of Physical Chemistry C, Vol.114, No.15 (April 2010) pp. 7205-7212, ISSN 1932-7447

Qin, Q.; Tao, J. \& Yang, Y. (2010). Preparation and Characterization of Polyaniline Film on Stainless Steel by Electrochemical Polymerization as a Counter Electrode of DSSC, Synthetic Metals, Vol.160, No.11-12, (June 2010) pp.1167-1172, ISSN 0379-6779

Runge, E.; Gross, E. K. U. (1984). Density-Functional Theory for Time-Dependent Systems. Physical Review Letter, Vol.52, No.12, (1984) pp.997-1000, ISSN 0031-9007

Soler, J. M.; Artacho, E.; Gale, J. D.; Garcia, A.; Junquera, J.; Ordejon, P. \& Sanchez-Portal, D. (2002). The SIESTA Method for Ab Initio Order-N Materials Simulation, Journal of Physics: Condensed Matter, Vol.14, No.11, (March 2002) pp. 2745-2779, ISSN 09538984

Xu, M.; Wenger, S.; Bala, H.; Shi, D.; Li, R.; Zhou, Y.; Zakeeruddin, S. M.; Grätzel, M. \& Wang, P. (2009). Tuning the Energy Level of Organic Sensitizers for HighPerformance Dye-Sensitized Solar Cells, Journal of Physical Chemistry C, Vol. 113, No.7 (February 2009) pp.2966-2973, ISSN 1932-7447

Yanai, T.; Tew, D. P. \& Handy, N. C. (2004). A New Hybrid Exchange-Correlation Functional Using the Coulomb-Attenuating Method (CAM-B3LYP), Chemical Physics Letter, Vol. 393, No.1-3 (July 2004) pp. 51-57, ISSN 0009-2614

Yum, J. H.; Hagberg, D. P.; Moon, S. J.; Karlsson, K. M.; Marinado, T.; Sun, L.; Hagfeldt, A.; Nazeeruddin, M. K \& Grätzel, M. (2009). Panchromatic Response in Solid-State Dye-Sensitized Solar Cells Containing Phosphorescent Energy Relay Dyes, Angewandte Chemie-International Edition, Vol. 48, No.49 (2009) pp. 1576-1580, ISSN1433-7851

Yu, Z.; Li, D.; Qin, D.; Sun, H.; Zhang, Y.; Luo, Y. \& Meng, Q. (2009). Research and Development of Dye-Sensitized Solar Cells, Materials China, Vol.28, No.7-8, (August 2009), pp. 7-15, ISSN 1674-3962

Zeng, W.; Cao, Y.; Bai, Y.; Wang, Y.; Shi, Y.; Zhang, M.; Wang, F.; Pan, C. \& Wang, P. (2010). Efficient Dye-Sensitized Solar Cells with an Organic Photosensitizer Ferturing Orderly Conjugated Ethylenedioxythiophene and Dithienosilole Blocks. Chemistry of Materials, Vol.22, No.5, (March 2010) pp. 1915-1925, ISSN 0897-4756 


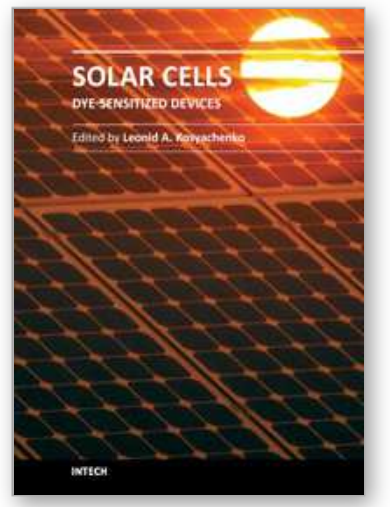

\author{
Solar Cells - Dye-Sensitized Devices \\ Edited by Prof. Leonid A. Kosyachenko
}

ISBN 978-953-307-735-2

Hard cover, 492 pages

Publisher InTech

Published online 09, November, 2011

Published in print edition November, 2011

The second book of the four-volume edition of "Solar cells" is devoted to dye-sensitized solar cells (DSSCs), which are considered to be extremely promising because they are made of low-cost materials with simple inexpensive manufacturing procedures and can be engineered into flexible sheets. DSSCs are emerged as a truly new class of energy conversion devices, which are representatives of the third generation solar technology. Mechanism of conversion of solar energy into electricity in these devices is quite peculiar. The achieved energy conversion efficiency in DSSCs is low, however, it has improved quickly in the last years. It is believed that DSSCs are still at the start of their development stage and will take a worthy place in the largescale production for the future.

\title{
How to reference
}

In order to correctly reference this scholarly work, feel free to copy and paste the following:

Yang Jiao, Fan Zhang and Sheng Meng (2011). Dye Sensitized Solar Cells Principles and New Design, Solar Cells - Dye-Sensitized Devices, Prof. Leonid A. Kosyachenko (Ed.), ISBN: 978-953-307-735-2, InTech, Available from: http://www.intechopen.com/books/solar-cells-dye-sensitized-devices/dye-sensitized-solar-cellsprinciples-and-new-design

\section{INTECH}

open science | open minds

\section{InTech Europe}

University Campus STeP Ri Slavka Krautzeka 83/A

51000 Rijeka, Croatia Phone: +385 (51) 770447

Fax: +385 (51) 686166

www.intechopen.com

\section{InTech China}

Unit 405, Office Block, Hotel Equatorial Shanghai

No.65, Yan An Road (West), Shanghai, 200040, China

中国上海市延安西路65号上海国际贵都大饭店办公楼405单元

Phone: +86-21-62489820

Fax: +86-21-62489821 
(C) 2011 The Author(s). Licensee IntechOpen. This is an open access article distributed under the terms of the Creative Commons Attribution 3.0 License, which permits unrestricted use, distribution, and reproduction in any medium, provided the original work is properly cited. 